\title{
Elementary Abelian $p$-groups of rank greater than or equal to $4 p-2$ are not CI-groups
}

\author{
Pablo Spiga
}

Received: 27 July 2006 / Accepted: 17 January 2007 /

Published online: 17 April 2007

(C) Springer Science+Business Media, LLC 2007

\begin{abstract}
In this paper we prove that an elementary Abelian $p$-group of rank $4 p-2$ is not a $\mathrm{CI}^{(2)}$-group, i.e. there exists a 2-closed transitive permutation group containing two non-conjugate regular elementary Abelian $p$-subgroups of rank $4 p-2$, see Hirasaka and Muzychuk (J. Comb. Theory Ser. A 94(2), 339-362, 2001). It was shown in Hirasaka and Muzychuk (loc cit) and Muzychuk (Discrete Math. 264(1-3), $167-185,2003)$ that this is related to the problem of determining whether an elementary Abelian $p$-group of rank $n$ is a CI-group.

As a strengthening of this result we prove that an elementary Abelian $p$-group $E$ of rank greater or equal to $4 p-2$ is not a CI-group, i.e. there exist two isomorphic Cayley digraphs over $E$ whose corresponding connection sets are not conjugate in Aut $E$.
\end{abstract}

Keywords Cayley graph · CI-group · Schur ring · 2-closure

\section{Introduction}

Let $H$ be a group and $S$ a subset of $H$. The Cayley digraph of $H$ with connection set $S$, denoted $\operatorname{Cay}(H, S)$, is the graph with vertex set $H$ and edge set $\left\{\left(h_{1}, h_{2}\right) \mid h_{1} h_{2}^{-1} \in S\right\}$. Two Cayley digraphs $\operatorname{Cay}(H, S)$ and $\operatorname{Cay}(H, T)$ are said to be Cayley isomorphic if there exists an element $g \in$ Aut $H$ such that $S^{g}=T$. A subset $S$ of a group $H$ is said to be a CI-subset (or Cayley isomorphic subset) if for each $T \subseteq H$ the digraphs $\operatorname{Cay}(H, S)$ and $\operatorname{Cay}(H, T)$ are isomorphic if and only if they are Cayley isomorphic. Finally, a group $H$ is said to be a CI-group if each subset of

This research was supported by a fellowship from the Pacific Institute for the Mathematical Sciences.

\section{P. Spiga $(\varangle)$}

Department of Mathematics and Computer Science, University of Lethbridge, 4401 University

Drive, Lethbridge, AB, Canada

e-mail: spiga@cs.uleth.ca 
$H$ is a CI-subset. We refer the interested reader to the survey article [5] for details, examples and the main results on CI-groups. We remark that the classification of CIgroups is not known and at the time of this writing it seems that the classification problem strongly depends on whether an elementary Abelian $p$-group of rank $n$ is a CI-group, see [5].

It was proved in [1] that $S \subseteq H$ is a CI-subset if and only if any regular subgroup of $\operatorname{Aut}(\operatorname{Cay}(H, S))$ isomorphic to $H$ is conjugate to $H$ in $\operatorname{Aut}(\operatorname{Cay}(H, S))$. This result is the starting point of most of the results on CI-groups.

In [3] the authors proved that if $V$ is a regular elementary Abelian $p$-subgroup of rank $n(n \leq 4, p>2)$ of a 2-closed permutation group $G$, then any regular subgroup $W$ of $G$ isomorphic to $V$ is conjugate to $V$ in $G$. In particular, by the former paragraph, as the automorphism group of a digraph is a 2-closed group we have that an elementary Abelian $p$-group of rank less than or equal to 4 is a CI-group. Motivated by this result the authors of [3] gave the following definition, see [3] page 341.

Definition 1 The group $H$ is said to be a $\mathrm{CI}^{(2)}$-group if for any 2-closed permutation group $G$ containing the right regular permutation representation of $H$, we have that any two regular subgroups of $G$ isomorphic to $H$ are conjugate in $G$.

Clearly if $H$ is a $\mathrm{CI}^{(2)}$-group, then $H$ is a CI-group. The authors of [3] asked for a complete classification of the $\mathrm{CI}^{(2)}$-elementary Abelian $p$-groups. At the time of this writing it is known that if an elementary Abelian $p$-group of rank $n$ is a $\mathrm{CI}^{(2)}$-group, then $n<2 p-1+\left(\begin{array}{c}2 p-1 \\ p\end{array}\right)$, see [6], and, if $n \leq 4$, then an elementary Abelian $p$-group of rank $n$ is a $\mathrm{CI}^{(2)}$-group, see [3].

In Sect. 3 we prove the following theorem.

Theorem 1 An elementary Abelian p-group of rank $n \geq 4 p-2$ is not a $\mathrm{CI}^{(2)}$-group.

We remark that a proof of Theorem 1 could be given using cohomological arguments. As a matter of consistency we present a proof closely related to the arguments in [6].

Finally, in Sect. 4, we prove Theorem 2, which is the main result of this paper.

Theorem 2 An elementary Abelian p-group of rank $n$ is not a CI-group if $n \geq 4 p-2$.

We remark that although Theorem 1 is a corollary of Theorem 2, the proof of the former is needed in order to prove the latter.

We refer to the precious book [9] for the main results on Schur rings and to [3] and [6] for their connections to $\mathrm{CI}^{(2)}$-groups and for the notation. We strongly advise the reader to use [6] as a crib.

\section{Preliminaries}

Let $p$ be a prime number, let $V, W$ be non-trivial elementary Abelian $p$-groups and let $U=V \mathrm{wr}_{W} W$ be the wreath product of $V$ with $W$ acting on $\Omega=W \times V$ in its natural imprimitive action. We recall that $(x, y)^{w f}=(x+w, y+f(x+w))$ for any 
$(x, y) \in \Omega$ and $w f \in U$. We denote by $B=V^{W}$ the base group of $U$. We denote by $\xi_{m}(U)$ then $m$ th term of the upper central series of $U$. In particular, the centre $\xi_{1}(U)$ of $U$ consists of the constant functions of $B$. We shall often identify a constant function of $B$, i.e. an element of $\xi_{1}(U)$, with its image. Let $G$ be a subgroup of $U$ containing $W \xi_{1}(U)$. Note that $W \xi_{1}(U)$ is an elementary Abelian regular subgroup of $U$. Furthermore $G=W L$ where $L=G \cap B$. We denote by $L_{0}$ the stabilizer in $G$ of the element $(0,0)$ of $\Omega$. The group $L_{0}$ defines a map $H: W \rightarrow 2^{V}=\{S \mid S \subseteq V\}$ by $H(w)=\left\{f(w) \mid f \in L_{0}\right\}$.

Lemma $1 H$ satisfies the following properties:

(i) $H(w)$ is a subspace of $V$;

(ii) $H(0)=\{0\}$;

(iii) $H(\beta w)=H(w)$ for any $\beta \neq 0$ in $\mathbb{F}_{p}$;

(iv) $H\left(w_{1}+w_{2}\right) \subseteq H\left(w_{1}\right)+H\left(w_{2}\right)$ for any $w_{1}, w_{2} \in W$.

Proof $(i) L_{0}$ is an $\mathbb{F}_{p}$-vector subspace of $B$ so $H(w)$ is an $\mathbb{F}_{p}$-vector subspace of $V$. (ii) By definition of $L_{0}$ we get that $H(0)=\{0\}$.

(iii) Let $f$ be in $L_{0}$ and $w$ in $W$. If $\beta \in \mathbb{F}_{p}$, then $g_{\beta}=f^{-\beta w}-f(\beta w)$ (here we are identifying $f(\beta w) \in V$ with the constant function $x \mapsto f(\beta w))$ is an element in $L_{0}$ (in fact $\left.g_{\beta}(0)=0\right)$ and $g_{\beta}(w)=f((\beta+1) w)-f(\beta w)$. Thus $f(w), f(2 w)-$ $f(w), \ldots, f((p-1) w)-f((p-2) w)$ and $-f((p-1) w)$ are elements of $H(w)$. So $f(\beta w) \in H(w)$ for any $\beta \in \mathbb{F}_{p}$. In particular $H(\beta w) \subseteq H(w)$ for any $\beta \in \mathbb{F}_{p}$. If $\beta \neq 0$, we have $H(w)=H(\beta w)$.

(iv) Now let $f$ be in $L_{0}$ and $w_{1}, w_{2} \in W$. Consider $g=f^{-w_{2}}-f\left(w_{2}\right)$ (as usual $f\left(w_{2}\right)$ denotes the element of $\xi_{1}(U)$ with image $\left.f\left(w_{2}\right)\right)$. Now $g \in L$ and $g(0)=\left(f^{-w_{2}}-f\left(w_{2}\right)\right)(0)=f\left(w_{2}\right)-f\left(w_{2}\right)=0$, so $g \in L_{0}$. Moreover $g\left(w_{1}\right)=$ $f\left(w_{1}+w_{2}\right)-f\left(w_{2}\right)$, therefore $f\left(w_{1}+w_{2}\right)=g\left(w_{1}\right)+f\left(w_{2}\right) \in H\left(w_{1}\right)+H\left(w_{2}\right)$.

We denote by $\operatorname{Der}(W, L)$ the group of derivations from $W$ to $L$ and by $\operatorname{Inn}(W, L)$ the group of inner derivations, see [8].

Lemma 2 Any regular elementary Abelian subgroup of $G$ is conjugate to $W \xi_{1}(U)$ if and only if $\operatorname{Der}(W, L)=\operatorname{Hom}\left(W, \xi_{1}(U)\right)+\operatorname{Inn}(W, L)$.

Proof Let $\delta$ be an element in $\operatorname{Der}(W, L)$. Then $H=X_{\delta} \xi_{1}(U)$ is a regular elementary Abelian subgroup of $G$, where $X_{\delta}=\left\{w w^{\delta} \mid w \in W\right\}$. In particular if $H$ is conjugate to $W \xi_{1}(U)$, then there exists $l \in L$ such that $X_{\delta} \subseteq W^{l} \xi_{1}(U)$. In other words, for any $w \in W$ there exists a unique $c_{w} \in \xi_{1}(U)$ such that $w^{\delta}=[w, l]+c_{w}$. The map $c_{-}: w \mapsto c_{w}$ is an homomorphism of $W$ into $\xi_{1}(U)$. In fact

$$
\begin{aligned}
{\left[w_{1}, l\right]^{w_{2}}+\left[w_{2}, l\right]+c_{w_{1}}+c_{w_{2}} } & =\left(\left[w_{1}, l\right]+c_{w_{1}}\right)^{w_{2}}+\left[w_{2}, l\right]+c_{w_{2}} \\
& =\left(w_{1}^{\delta}\right)^{w_{2}}+w_{2}^{\delta} \\
& =\left(w_{1}+w_{2}\right)^{\delta}=\left[w_{1}+w_{2}, l\right]+c_{w_{1}+w_{2}}
\end{aligned}
$$

and so $c_{w_{1}+w_{2}}=c_{w_{1}}+c_{w_{2}}$. Thus $\delta=c_{-}+[-, l] \in \operatorname{Hom}\left(W, \xi_{1}(U)\right)+\operatorname{Inn}(W, L)$. 
As any regular elementary Abelian subgroup $H$ of $G$ is of the form $X_{\delta} \xi_{1}(U)$, for some $\delta \in \operatorname{Der}(W, L)$, the other side of the implication is totally trivial.

Assume that the group $G$ is 2-closed. Every orbital of $G$ corresponds to an orbit of the point stabilizer $L_{0}$, i.e. every orbital of $G$ corresponds to a suborbit of the form $(w, v)^{L_{0}}=\left\{(w, f(w)+v) \mid f \in L_{0}\right\}=(w, v+H(w))$. The group $G$ contains the regular subgroup $W \xi_{1}(U)$ and so every orbital is a Cayley digraph over $W \times V$. In particular the orbital corresponding to the suborbit $(w, v)^{L_{0}}$ is $\left\{\left(\left(w_{1}, v_{1}\right),\left(w_{2}, v_{2}\right)\right) \mid\right.$ $\left.\left(w_{1}-w_{2}, v_{1}-v_{2}\right) \in(w, v+H(w))\right\}=\operatorname{Cay}(W \times V,(w, v+H(w)))$. Furthermore $G=\bigcap_{(w, v)} \operatorname{Aut}\left(\Gamma_{(w, v)}\right)$, where $\Gamma_{(w, v)}=\operatorname{Cay}(W \times V,(w, v+H(w)))$.

Let us define the $\mathbb{F}_{p}$-vector space

$$
\begin{aligned}
\operatorname{Hom}_{H}(W, V)= & \left\{\psi \in B \mid \psi\left(w_{1}+w_{2}\right)-\psi\left(w_{1}\right)-\psi\left(w_{2}\right) \in H\left(w_{1}\right) \cap H\left(w_{2}\right)\right. \\
& \text { for any } \left.w_{1}, w_{2} \in W, \psi(0)=0\right\},
\end{aligned}
$$

see [6].

Next if $\delta \in \operatorname{Der}(W, L)$, we define $\theta(\delta): W \rightarrow V$ by $\theta(\delta)(w)=\left(w^{\delta}\right)(0)$.

Lemma $3 \theta$ is an injective homomorphism of $\operatorname{Der}(W, L)$ into $\operatorname{Hom}_{H}(W, V)$. If $G$ is 2-closed, then $\theta$ is an isomorphism.

Proof Clearly $\theta(\delta)(0)=\left(0^{\delta}\right)(0)=0$. Let $w_{1}, w_{2}$ be elements of $W$. Set $w_{1}^{\delta}=f+g$ where $f \in L_{0}$ and $g \in \xi_{1}(U)$. We have

$$
\begin{aligned}
\theta(\delta)\left(w_{1}+w_{2}\right)-\theta(\delta)\left(w_{1}\right)-\theta(\delta)\left(w_{2}\right) & =\left(w_{1}+w_{2}\right)^{\delta}(0)-w_{1}^{\delta}(0)-w_{2}^{\delta}(0) \\
& =\left(\left(w_{1}\right)^{\delta}\right)^{w_{2}}(0)+w_{2}^{\delta}(0)-w_{1}^{\delta}(0)-w_{2}^{\delta}(0) \\
& =w_{1}^{\delta}\left(-w_{2}\right)-w_{1}^{\delta}(0)=f\left(-w_{2}\right) \in H\left(w_{2}\right) .
\end{aligned}
$$

By symmetry (the group $W$ is commutative) we get that

$$
\theta(\delta)\left(w_{1}+w_{2}\right)-\theta(\delta)\left(w_{1}\right)-\theta(\delta)\left(w_{2}\right) \in H\left(w_{1}\right) .
$$

Therefore $\theta(\delta) \in \operatorname{Hom}_{H}(W, V)$.

The map $\theta$ is an homomorphism. If $\delta_{1}, \delta_{2} \in \operatorname{Der}(W, L)$ and $w \in W$, then $\theta\left(\delta_{1}+\right.$ $\left.\delta_{2}\right)(w)=w^{\delta_{1}+\delta_{2}}(0)=w^{\delta_{1}}(0)+w^{\delta_{2}}(0)=\left(\theta\left(\delta_{1}\right)+\theta\left(\delta_{2}\right)\right)(w)$. Moreover $\theta(0)=0$. Finally $w^{\alpha \delta}=\alpha w^{\delta}$ for any $\alpha \in \mathbb{F}_{p}$ and so $\theta(\alpha \delta)=\alpha \theta(\delta)$.

The map $\theta$ is injective. $\theta(\delta)=0$ if and only if $w^{\delta}(0)=0$ for any $w \in W$. So $w^{\delta} \in$ $L_{0}$ for any $w \in W$. In particular $0=\left(w+w_{1}\right)^{\delta}(0)=\left(\left(w^{\delta}\right)^{w_{1}}+w_{1}^{\delta}\right)(0)=w^{\delta}\left(-w_{1}\right)$ for any $w_{1}, w$. Thus $w^{\delta}=0$ for any $w$. Therefore $\delta=0$.

Assume that $G$ is 2-closed. Let $\psi$ be an element of $\operatorname{Hom}_{H}(W, V)$. Define $\eta(w)=$ $\psi(-w)$ for any $w \in W$. The function $\eta$ lies in the base group $B$. Furthermore $\eta$ lies in $\operatorname{Hom}_{H}(W, V)$, in fact $\eta(0)=\psi(0)=0$ and $\eta\left(w_{1}+w_{2}\right)-\eta\left(w_{1}\right)-\eta\left(w_{2}\right)=$ $\psi\left(-w_{1}-w_{2}\right)-\psi\left(-w_{1}\right)-\psi\left(-w_{2}\right) \in H\left(-w_{1}\right) \cap H\left(-w_{2}\right)=H\left(w_{1}\right) \cap H\left(w_{2}\right)$. We claim that $\eta$ normalizes $G$, i.e. $[W, \eta] \subseteq L$. Let $w^{\prime}$ be in $W$. We have to prove that $\left[w^{\prime}, \eta\right] \in L$, i.e. $g=\eta-\eta^{w^{\prime}}+\eta\left(-w^{\prime}\right) \in L_{0}$. We claim that $\Gamma_{(w, v)}^{g}=\Gamma_{(w, v)}$ for every 
$(w, v) \in \Omega$. Let $\left(\left(w_{1}, v_{1}\right),\left(w_{2}, v_{2}\right)\right)$ be in $\Gamma_{(w, v)}$. In particular $w_{1}-w_{2}=w$ and $v_{1}-v_{2}=v+x$ for some $x$ in $H(w)$. We have

$$
\left(\left(w_{1}, v_{1}\right),\left(w_{2}, v_{2}\right)\right)^{g}=\left(\left(w_{1}, v_{1}+g\left(w_{1}\right)\right),\left(w_{2}, v_{2}+g\left(w_{2}\right)\right)\right)
$$

and

$$
v_{1}+g\left(w_{1}\right)-\left(v_{2}+g\left(w_{2}\right)\right)=v+x+\eta\left(w_{1}\right)-\eta\left(w_{1}-w^{\prime}\right)-\eta\left(w_{2}\right)+\eta\left(w_{2}-w^{\prime}\right) .
$$

Now, the function $\eta$ lies in $\operatorname{Hom}_{H}(W, V)$, therefore

$$
\begin{array}{r}
\eta\left(w_{2}+w\right)-\eta\left(w_{2}\right)-\eta(w) \in H(w) \\
-\eta\left(w_{2}+w-w^{\prime}\right)+\eta\left(w_{2}-w^{\prime}\right)+\eta(w) \in H(w)
\end{array}
$$

so $\eta\left(w_{1}\right)-\eta\left(w_{1}-w^{\prime}\right)-\eta\left(w_{2}\right)+\eta\left(w_{2}-w^{\prime}\right) \in H(w)$. Hence $v_{1}+g\left(w_{1}\right)-$ $\left(v_{2}+g\left(w_{2}\right)\right) \in v+H(w)$. This yields $\Gamma_{(w, v)}^{g}=\Gamma_{(w, v)}$ for every $(w, v) \in \Omega$. So, $g \in G^{(2)}=G$. Therefore $[W, \eta] \subseteq L$ and $[-,(-\eta)] \in \operatorname{Der}(W, L)$. Finally $\theta([-,(-\eta)])(w)=[w,-\eta](0)=\left(\eta^{w}-\eta\right)(0)=\eta(-w)-\eta(0)=\psi(w)$, so $\theta([-,(-\eta)])=\psi$. This proves $\theta$ is surjective and $\operatorname{Der}(W, L) \cong \operatorname{Hom}_{H}(W, V)$.

Lemma 4 Assume that $G$ is a 2-closed group. Any two regular elementary Abelian subgroups of $G$ are conjugate if and only if for any $\psi \in \operatorname{Hom}_{H}(W, V)$ there exists $\Lambda \in \operatorname{Hom}(W, V)$ such that $(\psi-\Lambda)(w) \in H(w)$ for every $w \in W$.

Proof Assume that any two regular elementary Abelian subgroups of $G$ are conjugate and let $\psi \in \operatorname{Hom}_{H}(W, V)$. So, by Lemmas 2 and 3 we have $\psi=\theta([-, l]+\Lambda)$ for some $l \in L_{0}$ and $\Lambda \in \operatorname{Hom}\left(W, \xi_{1}(U)\right)$. Clearly

$$
\begin{aligned}
(\theta([-, l]+\Lambda)-\Lambda)(w) & =w^{[-, l]+\Lambda}(0)-\Lambda(w) \\
& =[w, l](0)=(-l)^{w}(0)=(-l)(-w) \in H(w)
\end{aligned}
$$

for any $w \in W$. Conversely, by Lemma 3 it is enough to prove that $\operatorname{Der}(W, L)=$ $\operatorname{Hom}\left(W, \xi_{1}(U)\right)+\operatorname{Inn}(W, L)$. Let $\delta$ be an element of $\operatorname{Der}(W, L)$. By hypothesis there exists $\Lambda \in \operatorname{Hom}(W, V)$ such that $(\theta(\delta)-\Lambda)(w) \in H(w)$ for any $w \in W$. Set $g(w)=$ $(\theta(\delta)-\Lambda)(-w)$ for any $w \in W$. Clearly $\Gamma_{(w, v)}^{g}=\Gamma_{(w, v+g(w))}=\Gamma_{(w, v)}$ for any $(w, v) \in \Omega$. This yields $g \in L$. We leave the reader to check that $\delta=[-,(-g)]+\Lambda$. Thus the proof is complete.

Summing up so far, a 2-closed subgroup $G$ of $U$ contains two non-conjugate elementary Abelian regular subgroups if and only if there exists $\psi \in \operatorname{Hom}_{H}(W, V)$ such that there does not exist $\Lambda \in \operatorname{Hom}(W, V)$ such that $(\psi-\Lambda)(w) \in H(w)$ for every $w \in W$, see Proposition 7 in [6]. Note that this description is based on the knowledge of $H$ and in particular the group $G$ does not appear.

Conversely, we show that a map $H$ satisfying $(i),(i i),(i i i),(i v)$ is intimately related to a 2-closed subgroup of $U$. For $(w, v) \in W \times V$ define $\Gamma_{(w, v)}=$ $\left\{\left(\left(w_{1}, v_{1}\right),\left(w_{2}, v_{2}\right)\right) \mid w_{1}-w_{2}=w, v_{1}-v_{2} \in H(w)+v\right\}$. Finally define, only 
for the remainder of this section, $A=\bigcap_{(w, v)} \operatorname{Aut}\left(\Gamma_{(w, v)}\right)$. Note that $W \times V$ acting via right multiplication is a regular subgroup of $\operatorname{Aut}\left(\Gamma_{(w, v)}\right)$. In particular $\Gamma_{(w, v)}=\operatorname{Cay}(W \times V,(w, v+H(w)))$.

We claim that $A$ is a subgroup of $U$. Firstly we prove that $\Sigma=\{\{w\} \times V\}_{w \in W}$ is a block system for $A$. Let $g$ be an element of $A$ and $\Delta$ be $\{w\} \times V$. Assume that $\Delta^{g} \cap \Delta \neq \emptyset$. So, $\left(w, v_{1}\right)^{g}=\left(w, v_{2}\right)$ for some $v_{1}, v_{2} \in V$. Let $(w, v) \in$ $\Delta$. Then $\left((w, v),\left(w, v_{1}\right)\right) \in \Gamma_{\left(0, v-v_{1}\right)}$ so $\left((w, v),\left(w, v_{1}\right)\right)^{g}=\left((w, v)^{g},\left(w, v_{2}\right)\right) \in$ $\Gamma_{\left(0, v-v_{1}\right)}$. This implies that $(w, v)^{g} \in \Delta$, so $\Delta^{g}=\Delta$. Next, we prove that $A$ acts regularly on $\Sigma$. The group $A$ is transitive on $\Omega$ and so $A$ is transitive on $\Sigma$. Assume that $g$ fixes $\{w\} \times V$ and let $w_{1} \in W$. Now, $(w, 0)^{g}=(w, v)$ for some $v \in V$ and $\left(w_{1}, 0\right)^{g}=\left(w_{2}, v_{2}\right)$ for some $w_{2} \in W$ and $v_{2} \in V$. Now $g \in \operatorname{Aut}\left(\Gamma_{\left(w_{1}-w, 0\right)}\right)$ and $\left(\left(w_{1}, 0\right),(w, 0)\right) \in \Gamma_{\left(w_{1}-w, 0\right)}$ so $\left(\left(w_{1}, 0\right)^{g},(w, 0)^{g}\right)=$ $\left(\left(w_{2}, v_{2}\right),(w, v)\right) \in \Gamma_{\left(w_{1}-w, 0\right)}$, so, $w_{2}-w=w_{1}-w$. Thus $w_{1}=w_{2}$. This says that $A$ acts regularly on $\Sigma$. Let us denote by $L$ the kernel of the permutation representation of $A$ on $\Sigma$ (i.e. the stabilizer of the set $\left\{0_{W}\right\} \times V$ ). It remains to prove that $L$ acts regularly on $\left\{0_{W}\right\} \times V$. The group $L$ contains $\left\{0_{W}\right\} \times V$ and so $L$ acts transitively on each set of the form $\{w\} \times V$. Let $g$ be in $L$. Assume that $(0,0)^{g}=(0,0)$. Let $v$ be in $V$ and let us prove that $(0, v)^{g}=(0, v)$. Note that $((0, v),(0,0)) \in \Gamma_{(0, v)}$ and so $((0, v),(0,0))^{g}=\left(\left(0, v_{1}\right),(0,0)\right)$ is an element of $\Gamma_{(0, v)}=\operatorname{Cay}(W \times V,(0, v))$, so, $v_{1}=v$. This proves that $A$ is a subgroup of $U$.

\section{The construction}

Since the elementary Abelian 2-group of rank 6 is known not to be a CI-group, see [7], we assume that $p \geq 3$.

We strongly use the well-known structure of the upper central series of the group $U$, see [4].

Let $V$ be the Galois field $\mathbb{F}_{p^{n}}$, where $n=2 p-1$, and let $\varepsilon_{1}, \ldots, \varepsilon_{n}$ be an $\mathbb{F}_{p}$-basis of $V$. Also, let $W$ be an $\mathbb{F}_{p}$-vector space of dimension $n$ with basis $e_{1}, \ldots, e_{n}$ and dual basis $e_{1}^{*}, \ldots, e_{n}^{*}$. Let $\mathcal{S}$ be the symmetric $V$-algebra on $e_{1}^{*}, \ldots, e_{n}^{*}$ and natural filtration $\mathcal{S}=\oplus_{m \in \mathbb{N}} \mathcal{S}_{m}$ (recall that $\mathcal{S}_{m}$ is spanned by the monomials $e_{i_{1}}^{* j_{1}} \otimes \cdots \otimes$ $e_{i_{k}}^{* j_{k}}$ of degree $m$ ). Let $\mathcal{M}_{m}$ be the $V$-subspace of $\mathcal{S}_{m}$ spanned by the monomials $e_{i_{1}}^{* j_{1}} \otimes \cdots \otimes e_{i_{k}}^{* j_{k}}$ of degree $m$ such that $j_{1}, \ldots, j_{k} \leq p-1$. Let $\pi: \mathcal{S} \rightarrow B=V^{W}$ be the valuation map and $Z_{i}=\pi\left(\mathcal{M}_{i}\right)$. For instance $\pi\left(e_{i_{1}}^{* j_{1}} \otimes \cdots \otimes e_{i_{k}}^{* j_{k}}\right)(w)$ is the element of $V$ defined by $\left(e_{i_{1}}^{*}(w)\right)^{j_{1}} \cdots\left(e_{i_{k}}^{*}(w)\right)^{j_{k}}$ for any $w \in W$. It is known that $B \cap \xi_{m}(U)=\oplus_{i \leq m-1} Z_{i}$ and that $\pi_{\mid \mathcal{M}_{i}}$ is injective. Without loss of generality we identify $e_{i_{1}}^{* j_{1}} \otimes \cdots \otimes e_{i_{k}}^{* j_{k}}$ in $\mathcal{M}_{m}$ with its image under $\pi$.

Let $X$ be the set $\{1, \ldots, n\}$ and $X_{i}=\{A \subseteq X|| A \mid=i\}$ for $i=0, \ldots, p$. If $A$ is a subset of $X$, then we denote by $e_{A}^{*}$ the element of $B$ defined by $\otimes_{i \in A} e_{i}^{*}$. Similarly $\varepsilon_{A}$ denotes $\sum_{i \in A} \varepsilon_{i} \in V$. We let $\varepsilon$ and $e$ denote $\varepsilon_{X}$ and $\sum_{i \in X} e_{i}$, respectively.

Consider

$$
f=\sum_{A \in X_{p}} \varepsilon_{X \backslash A} e_{A}^{*} \in B .
$$


Define $L=[W, f]$. If $w_{1}, w_{2} \in W$, then $\left[w_{1}+w_{2}, f\right]=\left[w_{1}, f\right]^{w_{2}}+\left[w_{2}, f\right]$. This shows that $G=\langle W, L\rangle=W L$. Since $[G, f]=[W, f] \subseteq G$, we have that $f$ normalizes $G$.

Let $C$ be a subset of $X$ of size $p-i$. Define

$$
g_{C}=\sum_{B \in X_{i}, C \cap B=\emptyset} \varepsilon_{X \backslash(B \cup C)} e_{B}^{*} .
$$

Lemma 5 The group $L$ is generated by the set $\left\{g_{C} \mid C \in X_{i}, 1 \leq i \leq p\right\}$ and $L_{0}$ is generated by $\left\{g_{C} \mid C \in X_{i}, 1 \leq i \leq p-1\right\}$.

Proof If $i \in A$, then $\left(e_{A}^{*}\right)^{e_{i}}(w)=\prod_{a \in A} e_{a}^{*}\left(w-e_{i}\right)=e_{A}^{*}(w)-e_{A \backslash\{i\}}^{*}(w)$ and so

$$
(\dagger) \quad\left[e_{i}, e_{A}^{*}\right]= \begin{cases}0 & \text { if } i \notin A, \\ e_{A \backslash\{i\}}^{*} & \text { if } i \in A .\end{cases}
$$

Using $(\dagger)$ we get

$$
\left[e_{i}, f\right]=\sum_{B \in X_{p-1}, i \notin B} \varepsilon_{X \backslash(B \cup\{i\})} e_{B}^{*}=g_{\{i\}} \in L \quad \text { for any } i \in X .
$$

More generally we have

$$
\left[e_{i}, g_{C}\right]= \begin{cases}0 & \text { if } i \in C, \\ g_{C \cup\{i\}} & \text { if } i \notin C .\end{cases}
$$

The group $L$ is generated by the left-normed commutators $\left[f, e_{i_{1}}, \ldots, e_{i_{k}}\right]$ for $k \geq 1$ and therefore $L$ is generated by $\left\{g_{C} \mid C \in X_{i}, 1 \leq i \leq p\right\}$. Our claim on $L_{0}$ is just an easy remark.

Corollary $1 G$ contains $\xi_{1}(U)$.

Proof By Lemma 5 we have that $L \cap \xi_{1}(U)$ is generated by $\left\{g_{C} \mid C \in X_{p}\right\}=\left\{\varepsilon_{X \backslash C} \mid\right.$ $\left.C \in X_{p}\right\}$. As usual we are identifying the elements of $V$ with the elements of $\xi_{1}(U)$. We leave the reader to show that $\left\{\varepsilon_{X \backslash C} \mid C \in X_{p}\right\}$ spans $V$. $G$.

By Corollary 1 we have that $U \supseteq G \supseteq W \xi_{1}(U)$ and so we can apply Section 2 to

Let $\varphi$ be the non-degenerate bilinear symmetric form on $V$ defined by $\varphi\left(\varepsilon_{i}, \varepsilon_{j}\right)=$ $\delta_{i j}$, where $\delta_{i j}$ is the Kronecker delta.

Lemma $6 H\left(e_{i}\right)=\varepsilon_{i}^{\perp}$ for any $i \in X$.

Proof We have that $g_{C}\left(e_{i}\right)=0$ for any $C \in X_{p-j}, j \geq 2$. Therefore, by Lemma 5, $H\left(e_{i}\right)=\left\langle g_{C}\left(e_{i}\right) \mid C \in X_{p-1}\right\rangle=\left\langle\varepsilon_{X \backslash(C \cup\{i\})} \mid i \notin C, C \in X_{p-1}\right\rangle$, while it is an easy exercise in linear algebra to prove that this vector space is $\varepsilon_{i}^{\perp}$.

Lemma 7 If $i \neq j$, then $H\left(e_{i}+e_{j}\right)=\left(\varepsilon_{i}-\varepsilon_{j}\right)^{\perp}$. 
Proof Arguing is the same way as in Lemma 6 we have $H\left(e_{i}+e_{j}\right)=$ $\left\langle g_{C}\left(e_{i}+e_{j}\right) \mid C \in X_{p-2} \cup X_{p-1}\right\rangle$. It is routine computation in linear algebra to prove that $\left\langle g_{C}\left(e_{i}+e_{j}\right) \mid C \in X_{p-2} \cup X_{p-1}\right\rangle=\left\langle\varepsilon_{X \backslash(C \cup\{i, j\})}, \varepsilon_{X \backslash\left(C^{\prime} \cup\{i\}\right)}+\varepsilon_{X \backslash\left(C^{\prime} \cup\{j\}\right)}\right| C \in$ $\left.X_{p-2}, C^{\prime} \in X_{p-1}, i, j \notin C \cup C^{\prime}\right\rangle=\left(\varepsilon_{i}-\varepsilon_{j}\right)^{\perp}$.

Lemma $8 H(e)=\varepsilon^{\perp}$.

Proof Let $C$ be a set of size $p-i$, where $1 \leq i \leq p-1$. Then

$$
\begin{aligned}
\varphi\left(g_{C}(e), \varepsilon\right) & =\sum_{B \in X_{i}, C \cap B=\emptyset} \varphi\left(\varepsilon_{X \backslash(B \cup C)}, \varepsilon\right)=\sum_{B \in X_{i}, C \cap B=\emptyset}(p-1) \\
& =-\left(\begin{array}{c}
p+i-1 \\
i
\end{array}\right)=0 .
\end{aligned}
$$

If $C \in X_{p-1}$, then we have $g_{C}(e)=\sum_{i \notin C} \varepsilon_{X \backslash(C \cup\{i\})}=-\varepsilon_{X \backslash C}$. Thus $\varepsilon^{\perp} \supseteq H(e) \supseteq$ $\left\langle g_{C}(e) \mid C \in X_{p-1}\right\rangle=\left\langle\varepsilon_{X \backslash C} \mid C \in X_{p-1}\right\rangle=\varepsilon^{\perp}$.

Lemma $9 H\left(e-e_{i}\right)=\left(\varepsilon+\varepsilon_{i}\right)^{\perp}$ for any $i \in X$.

Proof Let $C$ be a set of size $p-j$, where $1 \leq j \leq p-1$. If $i \notin C, B \in X_{j}$, $i \notin B, B \cap C=\emptyset$, then $\varphi\left(\varepsilon_{X \backslash(C \cup B)}, \varepsilon+\varepsilon_{i}\right)=0$. If $i \in C, B \in X_{j}, C \cap B=\emptyset$, then $\varphi\left(\varepsilon_{X \backslash(C \cup B)}, \varepsilon+\varepsilon_{i}\right)=p-1$. The function $e_{A}^{*}$ is not 0 on the element $e-e_{i}$ if and only if $i \notin A$. Thus

$$
\varphi\left(g_{C}\left(e-e_{i}\right), \varepsilon+\varepsilon_{i}\right)=\sum_{B \in X_{j}, i \notin B, C \cap B=\emptyset} \varphi\left(\varepsilon_{X \backslash(B \cup C)}, \varepsilon+\varepsilon_{i}\right)
$$

So, if $i \notin C$, then (*) is equal to 0 . If $i \in C$, then $(*)$ is equal to

$$
\sum_{B \in X_{j}, C \cap B=\emptyset}(p-1)=(p-1)\left(\begin{array}{c}
p+j-1 \\
j
\end{array}\right)=0 .
$$

If $C \in X_{p-1}$ and $i \notin C$, then $g_{C}\left(e-e_{i}\right)=\sum_{j \notin C, j \neq i} \varepsilon_{X \backslash(C \cup\{j\})}=-\varepsilon_{X \backslash C}-$ $\varepsilon_{X \backslash(C \cup\{i\})}$. If $C \in X_{p-1}$ and $i \in C$, then $g_{C}\left(e-e_{i}\right)=\sum_{j \notin C} \varepsilon_{X \backslash(C \cup\{j\})}=-\varepsilon_{X \backslash C}$. Now, $\left(\varepsilon+\varepsilon_{i}\right)^{\perp} \supseteq H\left(e-e_{i}\right) \supseteq\left\langle g_{C}\left(e-e_{i}\right) \mid C \in X_{p-1}\right\rangle=\left\langle\varepsilon_{X \backslash C}, \varepsilon_{X \backslash C^{\prime}}+\varepsilon_{X \backslash\left(C^{\prime} \cup\{i\}\right)}\right|$ $\left.C^{\prime}, C \in X_{p-1}, i \in C, i \notin C^{\prime}\right\rangle=\left(\varepsilon+\varepsilon_{i}\right)^{\perp}$.

Lemma $10 f\left(e_{i}\right)=0, f\left(e_{i}+e_{j}\right)=0, f(e)=\varepsilon, f\left(e-e_{i}\right)=\varepsilon$, for any $i, j \in X$, $i \neq j$.

Proof Clearly the first two identities are trivial. We have $f(e)=\sum_{A \in X_{p}} \varepsilon_{X \backslash A}=$ $\left(\begin{array}{c}2 p-2 \\ p\end{array}\right) \sum_{i \in X} \varepsilon_{i}=\varepsilon$. Finally $f\left(e-e_{i}\right)=\sum_{A \in X, i \notin A} \varepsilon_{X \backslash A}=\left(\begin{array}{c}2 p-3 \\ p\end{array}\right) \sum_{j \in X, j \neq i} \varepsilon_{j}+$ $\left(\begin{array}{c}2 p-2 \\ p\end{array}\right) \varepsilon_{i}=\varepsilon$.

Springer 
Let $g$ be the linear map defined by

$$
2^{-1} \sum_{i \in X} \varepsilon_{X \backslash\{i\}} e_{i}^{*}
$$

We have $g\left(e_{i}\right)=2^{-1} \varepsilon_{X \backslash\{i\}}, \quad g\left(e_{i}+e_{j}\right)=2^{-1}\left(\varepsilon_{X \backslash\{i\}}+\varepsilon_{X \backslash\{j\}}\right), \quad g(e)=$ $2^{-1} \sum_{j \in X} \varepsilon_{X \backslash\{j\}}=-\varepsilon$ and $g\left(e-e_{i}\right)=-\varepsilon-2^{-1} \varepsilon_{X \backslash\{i\}}$.

Lemma 11 There exists no linear map $\Lambda: W \rightarrow V$ such that $(f-\Lambda)(w) \in H(w)$ for every $w \in W$.

Proof By the preliminary remark on the map $g$ and by Lemmas 6, 7, 8, 9, 10 we have that $(f+g)\left(e_{i}\right) \in H\left(e_{i}\right),(f+g)\left(e_{i}+e_{j}\right) \in H\left(e_{i}+e_{j}\right),(f+g)(e) \in H(e)$. Therefore it suffices to prove that there exists no linear map $x$ such that $x\left(e_{i}\right) \in \varepsilon_{i}^{\perp}$, $x\left(e_{i}+e_{j}\right) \in\left(\varepsilon_{i}-\varepsilon_{j}\right)^{\perp}, x(e) \in \varepsilon^{\perp}$ and $\varphi\left(x\left(e-e_{n}\right)-2^{-1} \varepsilon_{X \backslash\{n\}}, \varepsilon+\varepsilon_{n}\right)=0$. By way of contradiction let us assume that we have such an $x=\sum_{i \in X} a_{i} e_{i}^{*}$, for $a_{i} \in V$.

In particular we have $\varphi\left(a_{j}, \varepsilon_{i}\right)=\varphi\left(a_{i}, \varepsilon_{j}\right)$ for any $i \neq j$ and $\varphi\left(a_{i}, \varepsilon_{i}\right)=0$. This yields

$$
\varphi\left(x(e), \varepsilon_{i}\right)=\sum_{j \in X} \varphi\left(a_{j}, \varepsilon_{i}\right)=\sum_{j \in X} \varphi\left(a_{i}, \varepsilon_{j}\right)=\varphi\left(a_{i}, \varepsilon\right) .
$$

Furthermore

$$
\varphi\left(x\left(e-e_{n}\right), \varepsilon+\varepsilon_{n}\right)=\varphi(x(e), \varepsilon)+\varphi\left(x(e), \varepsilon_{n}\right)-\varphi\left(a_{n}, \varepsilon\right)-\varphi\left(a_{n}, \varepsilon_{n}\right)=0 .
$$

So $\varphi\left(x\left(e-e_{n}\right)-2^{-1} \varepsilon_{X \backslash\{n\}}, \varepsilon+\varepsilon_{n}\right)=-2^{-1} \varphi\left(\varepsilon_{X \backslash\{n\}}, \varepsilon+\varepsilon_{n}\right)=1 \neq 0$, a contradiction. Thus the result is proved.

Theorem 1 An elementary Abelian p-group of rank $n \geq 4 p-2$ is not a $\mathrm{CI}^{(2)}$-group.

Proof By construction the element $f$ normalizes the 2-closure $G^{(2)}$ of $G$. We have $G^{(2)}=W L^{(2)}$ and clearly $G$ and $G^{(2)}$ induce the same map $H$. Therefore $[-, f] \in$ $\operatorname{Der}(W, L)$ and $\theta([-, f]) \in \operatorname{Hom}_{H}(W, V)$. We have $\theta([-, f])(w)=[w, f](0)=$ $-f(-w)$. Thus $f \in \operatorname{Hom}_{H}(W, V)$. The group $W \times V$ has rank $4 p-2$. Therefore, by Lemma 4 and Lemma 11 , the group $W \times V$ is not a $\mathrm{CI}^{(2)}$-group. It is an easy application of the first paragraph of Sect. 5.1 in [5] to see that any elementary Abelian $p$-group of rank $n \geq 4 p-2$ is not a $\mathrm{CI}^{(2)}$-group.

Based on some computer evidence we present the following conjecture.

Conjecture The group $G$ is 2 -closed.

\section{Proof of Theorem 2}

As in Section 3, we assume that $p>2$. In this section we shall repetitively use Proposition 22.1 (Schur-Wielandt principle), Propositions 22.4, 23.5 and Theorem 23.9 
in [9]. For the computations inside the group algebra $\mathbb{Q}[W \times V]$ we shall stick to the notation and to the terminology of Sect. 2 and 4 in [6].

In the proof of Theorem 1 we have shown that $f \in \operatorname{Hom}_{H}(W, V)$. Moreover, it was proved in [6] (Proposition 5 page 173) that the linear span, $\mathcal{A}_{H}$, of the simple quantities $\{(w, H(w)+v)\}_{w \in W, v \in V}$ is a Schur ring.

Let $E$ be $\left\{e_{i}\right\}_{i \in X} \cup\left\{e_{i}+e_{j}\right\}_{i \neq j} \cup\left\{e, e-e_{n}\right\}$. Note that in the proof of Lemma 11 we proved that there exists no linear function $\Lambda$ such that $(f-\Lambda)(w) \in H(w)$ for every $w \in E$.

Proposition 1 If $S$ is an $\mathcal{A}_{H}$-subset such that

$$
\underline{(w, H(w))} \in\langle\langle\underline{S}\rangle\rangle \quad \text { for every } w \in E,
$$

then $S$ is not a CI-subset of $W \times V$. In particular $W \times V$ is not a CI-group $(\langle\langle\underline{S}\rangle\rangle$ denotes the Schur ring generated by $\underline{S}$ ).

Proof The following argument mimics Proposition 4 in [6]. Assume $S$ is a CIsubset. Since $f \in \operatorname{Hom}_{H}(W, V)$, it is easy to check that $\operatorname{Cay}(W \times V, T)^{f}=$ Cay $\left(W \times V, T^{f}\right)$ for any simple quantity $T$ in $\mathcal{A}_{H}$ (see Proposition 6 in [6]). So, since $S$ is an $\mathcal{A}_{H}$-subset, we have $\operatorname{Cay}\left(W \times V, S^{f}\right)=\operatorname{Cay}(W \times V, S)^{f} \cong \operatorname{Cay}(W \times V, S)$. Therefore, since $S$ is a CI-subset, we get $S^{f}=S^{g}$ for some $g$ in $\operatorname{Aut}(W \times V)$. Then, $\operatorname{Cay}(W \times V, S)^{f}=\operatorname{Cay}\left(W \times V, S^{f}\right)=\operatorname{Cay}\left(W \times V, S^{g}\right)=\operatorname{Cay}(W \times V, S)^{g}$. Therefore $f g^{-1}$ is an automorphism of Cay $(W \times V, S)$.

Now, using Equation (1), the reader can verify (see Theorem 2.4 in [6]) that $f g^{-1}$ is an automorphism of $\operatorname{Cay}(W \times V,(w, H(w)))$ for every $w \in E$. Thus $(w, H(w))^{f}=(w, H(w))^{g}$ for every $w \in E$. Hence $(w, H(w))$ is a CI-subset for every $w \in E$ and

$$
[(w, H(w))]_{w \in E} \cong_{\text {Cay }}\left[(w, H(w))^{f}\right]_{w \in E} .
$$

Now, Proposition 7 in [6] yields that there exists $\Lambda \in \operatorname{Hom}(W, V)$ such that $(f-$ $\Lambda)(w) \in H(w)$ for every $w \in E$, a contradiction.

This proves that $S$ is not a CI-subset of $W \times V$. Therefore $W \times V$ is not a CIgroup.

Proof of Theorem 2 Like in Theorem 1, it is enough to prove that $W \times V$ is not a CI-group (see the first paragraph of Sect. 5.1 in [5]). Let $S$ be the $\mathcal{A}_{H}$-subset

$$
\begin{aligned}
& \left(\bigcup_{i \in X}\left(0, \varepsilon_{i}\right)\right) \bigcup\left(\bigcup_{i, j \in X, i \neq j}\left(e_{i}+e_{j}, H\left(e_{i}+e_{j}\right)\right)\right) \bigcup\left(e_{n}, H\left(e_{n}\right)\right) \\
& \bigcup\left(e-e_{n}, H\left(e-e_{n}\right)\right) \bigcup\left(\bigcup_{1 \leq j \leq p-1}\left(j e_{2 j}, H\left(e_{2 j}\right)\right)\right) \bigcup(e, H(e)) \\
& \bigcup\left(\bigcup_{1 \leq j \leq p-1}\left(j e_{2 j-1}, H\left(e_{2 j-1}\right)+\{ \pm \varepsilon\}\right)\right) .
\end{aligned}
$$


By Proposition 1, it remains to prove that Equation (1) holds for the set $S$. Let us denote by $\mathcal{A}$ the Schur ring $\langle\langle\underline{S}\rangle\rangle$.

Case $\mathbf{p} \geq \mathbf{5}$. The element $(\underline{S}+\underline{S}) \circ \underline{S}$ lies in $\mathcal{A}$ and it can be written as

$$
\sum_{(a, b) \in S} \lambda(a, b) \underline{(a, b)},
$$

where $\lambda(a, b)$ is the number of solutions of the equation

$$
(\ddagger) \quad(u, x)+(v, y)=(a, b)
$$

with $(u, x),(v, y) \in S$. It can be easily shown that

$$
\begin{aligned}
(\underline{S}+\underline{S}) \circ \underline{S}= & 0 T_{1} \uplus 2 p^{n-2} T_{2} \uplus 2(n-2) T_{3} \uplus\left(2(n-2) \uplus 2 p^{n-2}\right) T_{4} \\
& \uplus\left(2(n-2) \uplus 4 p^{n-2}\right) T_{5} \uplus 2(n-1) T_{6} \uplus\left(2(n-1) \uplus 6 p^{n-2}\right) T_{7}
\end{aligned}
$$

where

$$
\begin{aligned}
& T_{1}=\biguplus_{i \in X} \underline{\left(0, \varepsilon_{i}\right)} \uplus \underline{\left(e-e_{n}, H\left(e-e_{n}\right)\right)}, \\
& T_{2}=\underline{(e, H(e))}, \\
& T_{3}=\biguplus_{i<j,\{i, j\} \nsubseteq\{1,2, n\}} \frac{\left(e_{i}+e_{j}, H\left(e_{i}+e_{j}\right)\right),}{} \\
& T_{4}=\underline{\left(e_{2}+e_{n}, H\left(e_{2}+e_{n}\right)\right)}, \\
& T_{5}=\left(e_{1}+e_{2}, H\left(e_{1}+e_{2}\right)\right) \uplus\left(e_{1}+e_{n}, H\left(e_{1}+e_{n}\right)\right), \\
& T_{6}=\biguplus_{2 \leq j \leq p-1} \underline{\left(j e_{2 j}, H\left(e_{2 j}\right)\right)} \uplus \biguplus_{2 \leq j \leq p-1} \underline{\left(j e_{2 j-1}, H\left(e_{2 j-1}\right)+\{ \pm \varepsilon\}\right),} \\
& T_{7}=\underline{\left(e_{1}, H\left(e_{1}\right)+\{ \pm \varepsilon\}\right)} \uplus \underline{\left(e_{2}, H\left(e_{2}\right)\right)} \uplus \underline{\left(e_{n}, H\left(e_{n}\right)\right)} .
\end{aligned}
$$

For instance, if $a=0$, then ( $\ddagger)$ has no solution and so $\lambda(0, b)=0$. Also if $a=e_{n}$, then (†) has solutions with $u=0, e_{n}, e_{2 p-2}+e_{n},-e_{2 p-2}, e_{2 p-3}+e_{n},-e_{2 p-3}$. Studying all this possibilities we get $\lambda(a, b)=2(n-1) \uplus 6 p^{n-2}$. All the other computations are similar.

Note that $0,2 p^{n-2}, 2(n-2),\left(2(n-2)+2 p^{n-2}\right),\left(2(n-2)+4 p^{n-2}\right), 2(n-1)$, $\left(2(n-1)+6 p^{n-2}\right)$ are all distinct. So, by Schur-Wielandt principle, $T_{1}, \ldots, T_{7}$ lie in $\mathcal{A}$.

If $i, j \neq n$, then $H\left(e-e_{n}\right)+\varepsilon_{i}=H\left(e-e_{n}\right)+\varepsilon_{j}$. Thus

$$
\begin{aligned}
\left(\biguplus_{i \in X} \underline{\left(0, \varepsilon_{i}\right)}\right)+\underline{\left(e-e_{n}, H\left(e-e_{n}\right)\right)}= & \biguplus_{i \in X} \underline{\left(e-e_{n}, H\left(e-e_{n}\right)+\varepsilon_{i}\right)} \\
= & (n-1) \underline{\left(e-e_{n}, H\left(e-e_{n}\right)+\varepsilon_{1}\right)} \\
& \biguplus \underline{\left(e-e_{n}, H\left(e-e_{n}\right)+\varepsilon_{n}\right)} .
\end{aligned}
$$


Therefore, we have

$$
\begin{aligned}
T_{1}+T_{1}= & \biguplus_{i \in X} \underline{\left(0,2 \varepsilon_{i}\right)} \uplus 2 \biguplus_{i<j} \underline{\left(0, \varepsilon_{i}+\varepsilon_{j}\right)} \uplus 2(n-1) \underline{\left(e-e_{n}, H\left(e-e_{n}\right)+\varepsilon_{1}\right)} \\
& \uplus 2 \underline{\left(e-e_{n}, H\left(e-e_{n}\right)+\varepsilon_{n}\right)} \uplus p^{n-1} \underline{\left(2\left(e-e_{n}\right), H\left(e-e_{n}\right)\right)} .
\end{aligned}
$$

By Schur-Wielandt principle $t=\left(2\left(e-e_{n}\right), H\left(e-e_{n}\right)\right)$ is an element of $\mathcal{A}$ and so,

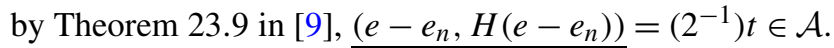

Note that $\left.(e, H(e))+\overline{\left(-e+e_{n}, H\left(e-e_{n}\right)\right.}\right)=\left(e_{n}, V\right)$ is a set of $\mathcal{A}$, so $\underline{\left(e_{n}, V\right)} \circ \underline{S}=$ $\left(e_{n}, H\left(e_{n}\right)\right) \in \mathcal{A}$.

Similarly, $\left(e_{2}+e_{n}, H\left(e_{2}+e_{n}\right)\right)+\left(-e_{n}, H\left(e_{n}\right)\right)=\left(e_{2}, V\right)$ is a set of $\mathcal{A}$, so $\underline{\left(e_{2}, H\left(e_{2}\right)\right)}=\underline{\left(e_{2}, V\right)} \circ \underline{S} \in \mathcal{A}$.

We have

$$
T_{6}+T_{6}=2 p^{n-2} U_{1} \uplus 8 p^{n-2} U_{2} \uplus 4 p^{n-2} U_{3} \uplus 2 p^{n-1} U_{4} \uplus p^{n-1} U_{5}
$$

where

$$
\begin{aligned}
& U_{1}=\biguplus_{2 \leq j_{1}<j_{2} \leq p-1} \underline{\left(j_{1} e_{2 j_{1}}+j_{2} e_{2 j_{2}}, V\right)}, \\
& U_{2}=\biguplus_{2 \leq j_{1}<j_{2} \leq p-1} \underline{\left(j_{1} e_{2 j_{1}-1}+j_{2} e_{2 j_{2}-1}, V\right)}, \\
& U_{3}=\biguplus_{2 \leq j_{2} \leq j_{1} \leq p-1} \underline{\left(j_{1} e_{2 j_{1}}+j_{2} e_{2 j_{2}-1}, V\right)}, \\
& U_{4}=\biguplus_{1 \leq j \leq p-1} \underline{\left(2 j e_{2 j-1}, H\left(e_{2 j-1}\right)\right)}, \\
& U_{5}=\biguplus_{2 \leq j \leq p-1} \underline{\left(2 j e_{2 j}, H\left(e_{2 j}\right)\right)} \uplus \biguplus_{2 \leq j \leq p-1} \underline{\left(2 j e_{2 j-1}, H\left(e_{2 j-1}\right)+\{ \pm 2 \varepsilon\}\right) .}
\end{aligned}
$$

The elements $2 p^{n-2}, 8 p^{n-2}, 4 p^{n-2}, 2 p^{n-1}, p^{n-1}$ are all distinct and so using SchurWielandt principle we have that $U_{1}, \ldots, U_{5}$ lie in $\mathcal{A}$.

We have

$$
F_{l}=\underline{\left(e_{2 l-1}+e_{2 l}, H\left(e_{2 l-1}+e_{2 l}\right)\right)}=\left(l^{-1}\right)\left(U_{3} \circ(l) T_{3}\right) \in \mathcal{A},
$$

for $2 \leq l \leq p-1$.

We have

$$
\left(U_{4}+(-2 l) F_{l}\right) \circ((-2) \underline{S})=p^{n-2} \underline{\left(-2 l e_{2 l}, H\left(e_{2 l}\right)\right)} \in \mathcal{A},
$$

for every $2 \leq l \leq p-1$. So, $\left(e_{2 l}, H\left(e_{2 l}\right)\right) \in \mathcal{A}$ for $2 \leq l \leq p-1$.

Now $\left(e_{2 l-1}+e_{2 l}, H\left(e_{2 l} \overline{\left.\left.+e_{2 l-1}\right)\right)+}\left(-e_{2 l}, H\left(e_{2 l}\right)\right)=\left(e_{2 l-1}, V\right)\right.\right.$ is a set of $\mathcal{A}$ for $2 \leq l \leq p-1$. Therefore $\underline{\left(e_{2 l-1}, H\left(e_{2 l-1}\right)\right)}=\underline{\left(e_{2 l-1}, V\right)} \circ\left(\left((2 l)^{-1}\right) U_{4}\right) \in \mathcal{A}$ for $2 \leq l \leq p-1$. 
So far we have proved that $\left(e_{i}, H\left(e_{i}\right)\right) \in \mathcal{A}$ for $i \geq 2$. In particular, by the definition of $T_{7}$, we get $q=\left(e_{1}, H\left(e_{1}\right) \overline{+\{ \pm \varepsilon\}) \in \mathcal{A}}\right.$. We have

$$
q+q=2 p^{n-1} \underline{\left(2 e_{1}, H\left(e_{1}\right)\right)} \uplus p^{n-1} \underline{\left(2 e_{1}, H\left(e_{1}\right)+\{ \pm 2 \varepsilon\}\right) .}
$$

Therefore, arguing as usual, we get $\left(e_{1}, H\left(e_{1}\right)\right) \in \mathcal{A}$.

Now $\left(e_{i}, H\left(e_{i}\right)\right)+\left(e_{j}, H\left(e_{j}\right)\right)=\left(e_{i}+e_{j}, V\right)$ is a set of $\mathcal{A}$. So $\left(e_{i}+e_{j}, H\left(e_{i}+e_{j}\right)\right)=\left(e_{i}+e_{j}, V\right) \circ \underline{S} \in \mathcal{A}$ for any $i, j$ with $i \neq j$.

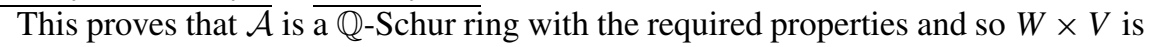
not a CI-group. Once again using the first paragraph of Sect. 5.1 in [5] we have that any elementary Abelian $p$-group of rank $n \geq 4 p-2$ is not a CI-group.

Case $\mathbf{p}=3$. We have

$$
\begin{aligned}
(\underline{S}+\underline{S}) \circ \underline{S}= & 0 T^{\prime} \uplus 2 p^{n-1} T^{*} \uplus 2 p^{n-2} T_{2} \uplus 2(n-2) T_{3} \uplus\left(2(n-2) \uplus 2 p^{n-2}\right) T_{4} \\
& \uplus\left(2(n-2)+4 p^{n-2}\right) T_{5} \uplus 2(n-1) T_{6} \uplus\left(2(n-1)+6 p^{n-2}\right) T_{7}
\end{aligned}
$$

where $T^{\prime}=\sum_{i \in X} \underline{\left(0, \varepsilon_{i}\right)}, T^{*}=\underline{\left(e-e_{n}, H\left(e-e_{n}\right)\right)}$ and $T_{2}, \ldots, T_{7}$ are defined as in the case $p \geq 5$.

Note that $0,2 p^{4}, 2 p^{n-2}, 2(n-2),\left(2(n-2)+2 p^{n-2}\right),\left(2(n-2)+4 p^{n-2}\right), 2(n-$ $1),\left(2(n-1)+6 p^{n-2}\right)$ are all distinct. So, by Schur-Wielandt principle, the elements $T^{\prime}, T^{*}, T_{2}, \ldots, T_{7}$ lie in $\mathcal{A}$. Now the computations are exactly the same as in the case $p \geq 5$.

Acknowledgements The author thanks Dr. Joy Morris for drawing his attention to the isomorphism problem of finite Cayley digraphs and for her many helpful suggestions during the preparation of this paper. Furthermore the author is in debt to the GAP group [2] and mainly to Prof. L.Soicher's package GRAPE.

\section{References}

1. Babai, L. (1977). Isomorphism problem for a class of point-symmetric structures. Acta Math. Acad. Sci. Hung., 29(3-4), 329-336.

2. The GAP Group (2005). GAP_-groups, algorithms, and programming, version 4.4, http://www. gap-system.org.

3. Hirasaka, M., \& Muzychuk, M. (2001). An elementary Abelian group of rank 4 is a CI-group. J. Comb. Theory Ser. A, 94(2), 339-362.

4. Lakatoš, P., \& Suščans'kiŭ, V. İ. (1976). The coincidence of central series in wreath products, I. Russian. Publ. Math. Debrecen, 23(1-2), 167-176.

5. Li, C. H. (2002). On Isomorphisms of finite Cayley graphs—a survey. Discrete Math., 256(1-2), 301334.

6. Muzychuk, M. (2003). An elementary Abelian group of large rank is not a CI-group. Discrete Math., 264(1-3), 167-185.

7. Nowitz, L. A. (1992). A non-Cayley-invariant Cayley graph of the elementary Abelian group of order 64. Discrete Math., 110(1-3), 223-228.

8. Robinson, D. J. S. (1980). A course in the theory of groups. Berlin: Springer (pp. 304-305).

9. Wielandt, H. (1964). Finite permutation groups. Berlin: Academic. 